\title{
Analysing the Impact of Pedestrian Network Centrality on Segment-Level Pedestrian Density
}

\author{
Daniel M. Pearce $\mathbb{D}^{1},{ }^{1}$ Ryoji Matsunaka ${ }^{D},{ }^{1}$ and Tetsuharu Oba $\mathbb{D}^{2}$ \\ ${ }^{1}$ Graduate School of Engineering, Kyoto University, Kyoto 615-8540, Japan \\ ${ }^{2}$ Graduate School of Management, Kyoto University, Kyoto 606-8501, Japan \\ Correspondence should be addressed to Daniel M. Pearce; dpearce@urban.kuciv.kyoto-u.ac.jp
}

Received 7 July 2021; Accepted 17 November 2021; Published 21 December 2021

Academic Editor: Rui Jiang

Copyright (C) 2021 Daniel M. Pearce et al. This is an open access article distributed under the Creative Commons Attribution License, which permits unrestricted use, distribution, and reproduction in any medium, provided the original work is properly cited.

\begin{abstract}
Studies have shown that street network centrality measures are capable of explaining a significant proportion of pedestrian activity. These studies typically employ street centreline networks that differ significantly from the networks that pedestrians use to traverse the built environment. Presently, centrality approaches are rarely applied to dedicated pedestrian network (DPNs). This creates uncertainty regarding their ability to explain pedestrian activity when derived from DPNs. This study addresses that gap by investigating the extent to which centrality metrics derived from DPNs can explain observed pedestrian densities, both alone and when controlling for other built environment variables in metro station environments in Asia. In total, four DPNs were created centred on metro stations in Bangkok, Manila, Osaka, and Taipei chosen to represent different urban typologies. Multivariate results show that centrality metrics alone explain a mere $6-24 \%$ of observed pedestrian densities when calculated on DPNs. When all factors are considered, the contribution of centrality remained consistent in most study sites but is somewhat reduced with land-use variables and proximity to rail transit revealed as the strongest predictors of pedestrian density. Pedestrian design factors were also frequently associated with pedestrian density. Finally, stronger associations between centrality and pedestrian densities were observed in the denser, more complex pedestrian environments. These findings provide insight into the performance of centrality measures applied to DPNs expanding pedestrian network research in this area.
\end{abstract}

\section{Introduction}

Pedestrians interact with the environment through a complex network of exclusive and shared paths that comprise the pedestrian network. These networks tie together all transportation modes and are fundamental to networkbased pedestrian studies [1]. As a result, their accessibility and connectivity are paramount not only in supporting walking but also in contributing to the overall efficiency of public transportation systems. Despite this fact, street centreline networks have instead been the default choice when calculating accessibility and connectivity and analysing the relationship between the built environment and walking [2].

Recent studies exploring the applicability of pedestrian networks suggest that they better represent how we interact with the built environment [1-5]. While street networks have performed well as proxies for pedestrian networks in most scenarios, studies conducting direct comparisons between accessibility and connectivity measures derived from both street and pedestrian networks have yielded mixed results. These studies argue that relying on street networks leads to distortions of reality, particularly regarding distance and route choice. For example, when utilising street networks, distance-based measures of accessibility are reportedly underestimated by as much as $7 \%$ in Asian cities [2] as well as destination accessibility by as much as $40 \%$ in some American neighbourhoods [3]. This is not surprising considering that street networks fail to account for the finergrained paths available to pedestrians that increase route options and directness. Additionally, non-distance-based measures of connectivity, such as the link-node ratio, have 
revealed counterintuitive results when switching to pedestrian networks, suggesting that suburban areas are more connected than traditionally gridded downtown areas [3]. These findings highlight the importance of network representation and the selection of metrics in pedestrian studies.

Presently, the vast majority of research investigating the suitability of pedestrian networks over street networks has focused on conventional approaches to accessibility and connectivity. These include applying common measures such as intersection density, percentage of four-way intersections, and the link-node ratio that have been shown to correlate strongly with pedestrian activity [4-6]. However, many of these measures have been criticised for providing a coarse account of connectivity across a general area. Hence, areas that score high in these measures fail to account for actual network permeability, obstructions to movement, and the spatial and structural pattern of street networks that define urban areas [7, 8]. Centrality approaches address these concerns and have been increasingly applied to expand research into accessibility and connectivity [9]. These approaches consider spatial structure by quantifying the importance of network elements in terms of how central they are in relation to other network elements [10]. Studies have shown that centrality measures derived from urban street networks are capable of explaining a significant proportion of pedestrian activity in a variety of different urban settings with minimal data requirements [8, 11-17].

Clearly, there is some debate as to the performance of accessibility and connectivity with pedestrian networks. This paper builds on existing studies by applying centrality approaches to dedicated pedestrian networks (DPNs). DPNs consist of all multilevel formal and informal pedestrian paths that pedestrians have legal access to, including sidewalks, pedestrian-only zones, shared streets, unmarked crossings, and paths through open spaces [2]. While centrality measures have been applied to street networks, they have rarely been applied to DPNs. This raises questions regarding the ability of these measures to explain pedestrian activity when using DPNs. Additionally, we argue that existing pedestrianoriented studies that utilise pedestrian networks have been conducted in different urban contexts outside of Asia, typically in North America and Australia, which have distinct urban morphologies and walking cultures. Therefore, the objectives of this research are (1) to investigate the extent to which common centrality metrics measured on DPNs can explain segment-level pedestrian densities, both alone and when controlling for other built environment variables, and (2) expand pedestrian network research in Asia. To achieve these aims, it is hoped that this research provides some clarity as to the potential of applying centrality measures to DPNs and encourages others to proceed further with research in this area.

\section{Literature Review}

Previous studies have confirmed that built environments which exhibit high population and employment densities, land-use diversity, street connectivity, and destination and transport accessibility are associated with increased levels of pedestrian activity $[6,18-24]$. In essence, these features encourage walking through improved connectivity and accessibility. This is achieved through mixing land uses, promoting smaller block sizes, and increasing intersection and street densities, among other methods [24-26]. This has the overall effect of increasing route options, reducing distance, and bringing destinations within closer proximity $[27,28]$. Importantly, environmental factors also play an important role in promoting pedestrian activity. These factors address both objective and subjective pedestrianoriented design features and encourage pedestrian activity by making walking not only more feasible but also more appealing to users. Common examples found to promote walking include the presence of pedestrian amenities, sidewalk continuity and width, crossing opportunities, and aesthetic design, including the attractiveness of the environment and presence and configuration of green spaces [29-35].

While a consensus has not been reached on the most important determinates of walking, research has consistently focused on street connectivity and by association accessibility, suggesting it as one of the most significant factors independent of other built environment features [9, 36-39]. Research has proliferated in this direction, though clear conclusions have not always emerged. This has been attributed to the coarse nature of average connectivity measures that have been applied in research and to the collinearity between land-use mix and street network design [8]. Centrality approaches address these issues through their ability to predict the proportion of pedestrian activity that can be attributed solely to the configuration of a street network, termed "natural movement" [40]. Street network configuration in turn is said to have an indirect effect on movement by influencing land-use distribution and density, which act as multipliers generating further movement $[40,41]$. While natural movement does not always quantify the largest proportion of pedestrian movement, it is argued to be the most consistent and pervasive type of movement [8]. Studies conducted in various built environments bolster this theory. The following sections will provide an overview of centrality approaches and applications of centrality to urban street networks in studies analysing pedestrian activity.

2.1. Centrality Approaches. Centrality approaches have appeared in the urban planning and design literature under various terms, including accessibility, proximity, integration, connectivity, and cost [42]. Regardless of the term, centrality approaches quantify the importance of network elements in terms of how central they are. The concept of centrality is multifaceted with different indices available depending on what the researcher's notion of "being central" is [10]. The two most common are betweenness, a measure of the importance of an element in a network in terms of how many shortest paths pass through it [43], and closeness, a measure of how close an element is to all other elements in a network calculated as the mean of the shortest path lengths [44]. These measures are typically encountered 
as choice and integration in studies that apply Space Syntax methods discussed below.

Two approaches have been applied in urban studiesthose based on graph theory and Space Syntax. These approaches differ primarily in how they represent network geometry. Graph approaches employ a "primal" network representation, where intersections are represented as nodes and streets as edges. Space Syntax reverses this geometry, placing streets at the centre of the analysis, creating a "dual" network representation [42]. This has traditionally resulted in different units being applied when measuring distance between network elements. Primal representations are the world standard for street network datasets utilised in transportation modelling which measure accessibility in terms of distance or cost [45]. In these applications, distance is measured metrically. Conversely, dual approaches have utilised various methods to represent street networks. The most common characteristic of which are axial lines, a generalisation model akin to lines of sight, and thus they are a measure of visual connectivity [14]. Axial lines are used to create axial maps where accessibility is measured in the number of topological "steps" or connections, and network elements are away from each other [9]. In recent years, Space Syntax has expanded to segmental analysis of either axial lines or GIS street networks, enabling the opportunity to measure distance between segments topologically, metrically, and angularly [11]. Angular-segmental approaches have proven particularly effective at predicting pedestrian movement $[46,47]$. Despite these advancements, topological approaches based on axial maps have remained the bedrock of Space Syntax research.

Both approaches have their pros and cons. Space Syntax offers analytical flexibility. However, numerous methods exist for generating axial lines that can introduce subjectivity into an analysis. Primal metric approaches that utilise existing standardised datasets are more objective and aid data processing. Porta et al. [42], for example, performed centrality analyses on four 1-square mile samples of urban street systems over primal and dual graphs and found that primal approach supported more comprehensive, objective, realistic results and a more feasible methodology for network analyses.

2.2. Centrality in Pedestrian Movement Studies. Studies have shown that street network centrality is capable of explaining a significant proportion of pedestrian activity [11, 17]. This research has largely been spearheaded by Space Syntax methods that utilise axial maps to conduct topographical analyses. The key metric in these studies is integration or closeness centrality, which has proven to be a powerful predictor of pedestrian activity. In short, these studies have shown that streets that are more "integrated" (or accessible within fewer direction changes) attract higher pedestrian numbers. For example, fundamental studies in London have found axial integration able to predict pedestrian activity in the range of $55-75 \%[12,15,40,48]$ with similar ranges also reported in other European capitals [49]. Betweenness centrality has also been shown to powerfully predict pedestrian activity with Law and Traunmueller's [17] results indicating that betweenness accounted for more than $50 \%$ of observed pedestrian movement in their London study sites.

Recent efforts by researchers drawing on the work of Space Syntax have applied other configurational measures derived from street centrelines, namely, metric reach, a measure of the total street length accessible within a specified radius of individual segments, and directional reach, a parametric measure of the total street length accessible within a certain number of direction changes defined by a threshold angle [50]. Studies using these measures have shown that the configuration of individual street elements within an area is significantly associated with walking $[14,28,50]$. In a study in Atlanta, USA, Özbil and Peponis [14] established strong correlations between metric reachnand directional reach and pedestrian movement densities in $1 \times 1$ mile study areas. Their results showed that these measures derived from street centrelines performed as well as integration derived from axial maps.

Centrality approaches have increasingly been applied to analyse relationships with pedestrian activity at the micro or segment scale. A number of these studies have conducted multivariate analyses controlling for key built environment variables in a variety of different urban settings $[8,11,16,28,51-53]$. Utilising pedestrian snapshot data, Fang et al. [16] conducted multivariate analyses between integration and several built environment characteristics, including store density, the overflow ratio of store-front space, density of building exits/entrances, building height, and store distance form block entrances on pedestrian densities within a mixed-use historic neighbourhood in Shanghai. Their results indicated the power of network structure in explaining observed pedestrian movement densities within local small-scale neighbourhoods that are more reminiscent of pedestrian paths. Among the four statistically significant variables, integration and store density were the largest predictors, explaining $35 \%$ and $27 \%$ of observed pedestrian densities, respectively.

Though centrality is positively associated with increased pedestrian activity, it is not always the most dominant factor. Recent research by Özbil et al. [8] further analysed the association between street network centrality in conjunction with land-use and street-level design variables such as sidewalk width and pedestrian flows in four Istanbul neighbourhoods. Separate multivariate models were constructed to better control for the effects of built environment variables, and separate multivariate models were constructed. Their results indicated that network connectivity, measured with integration and directional reach, is a significant predictor of pedestrian activity. However, across all study sites, the most significant predictor was accessibility to different groundfloor land uses that alone accounted for $38 \%$ of the variation in pedestrian densities. Importantly, although integration was not always significant, with the exception Küçükçekmece, the introduction of centrality measures added $12-20 \%$ to the predictive power of their models. Özer and Kubat [53] reported similar results between the number of commercial and service land uses and integration and pedestrian activity in a separate study conducted in Istanbul. 
2.3. Literature Contribution. The literature is clear that centrality measures derived from urban street networks are capable of explaining a substantial proportion of pedestrian activity. While these networks have performed well as a proxy for pedestrian networks, they fail to account for all paths available to pedestrians. To rectify this, some researchers have introduced missing pedestrian data to these networks $[16,17]$. In these applications, however, a generalised street network still takes precedence over dedicated pedestrian infrastructure. Accordingly, researchers have begun exploring the applicability of standalone pedestrian networks. In studies that directly compare accessibility and connectivity calculated on both pedestrian and street networks, substantial differences are reported with generally higher values observed in pedestrian networks [1-5]. Additionally, studies verifying the validity of pedestrian networks using pedestrian data are minimal. Several studies have begun exploring the relationship between pedestrian network connectivity and levels of physical activity with mixed results $[4,5]$. Yet, there are minimal cases of centrality being applied to true pedestrian networks $[10,54]$. This study fills this gap by utilising DPNs to analyse the relationship between centrality and segment-level pedestrian density. Finally, we conducted this study in Asian cities where pedestrian network research conducted in English is less developed.

\section{Materials and Methods}

The following section details study sites, data collection procedures, and our network approach and defines study variables for addressing our stated research objectives. This study uses some methods described in the work of Pearce et al. [2] and the methods' description partly reproduces their wording.

3.1. Study Sites. DPNs were constructed for four study sites located in Bangkok, Manila, Osaka, and Taipei. These cities were chosen to represent a variety of urban forms at different levels of development determined by the UN Human Development Index (HDI) [55]. Each study site consists of a 400 m Euclidean DPN centred on a major metro station, corresponding to a 5-minute walking catchment.

The following stations were selected: BangkokSukhumvit, Manila-Carriedo, Osaka-Namba, and TaipeiSongjiang Nanjing (Table 1 and Figure 1). These stations represent retail-led urban centres and were selected due to their high levels of ridership and pedestrian amenities. Bangkok-Sukhumvit has the least dense pedestrian network, characterised by long blocks and cul-de-sacs. It consists of a mixture of sidewalks, shared paths, and pedestrian walkways that provide limited crossing opportunities across major thoroughfares. Manila-Carriedo constitutes a deformed grid of varying block sizes. The area is dominated by a sprawling market east of the station. Sidewalks are present throughout the area but are usually of poor quality and are frequently blocked, causing pedestrians to mix with traffic. OsakaNamba is the densest and most complex pedestrian environment, consisting of shorter blocks, public spaces, pedestrian-only paths, and a large network of subsurface paths. Major streets are lined with sidewalks, while narrow streets are typically shared. Finally, Taipei-Songjiang Nanjing represents a gridiron network of regular rectangular blocks. Similar to Osaka, the pedestrian network is dense and is characterised by sidewalks that line major streets and a fine-grained network of narrow shared streets punctuated by public spaces.

3.2. Network Dataset. OpenStreetMap (OSM) network data were used to construct geographically accurate DPNs. DPNs are primal networks that consist of all multilevel formal and informal pedestrian facilities that pedestrians have legal access to. Thus, they consist of but are not limited to sidewalks, pedestrian-only zones, shared streets, unmarked crossings, and paths through open spaces (Figure 2). Network data were cross-referenced with aerial and satellite imagery provided by national agencies or Google Earth services to ensure the existence of each network link. Missing pedestrian links identified during this process were manually digitised in ArcGIS 10.7 according to the DPN principles detailed by Pearce et al. [2]. These principles prescribe modelling paths on all levels, indoor paths through large shopping centres and stations, and perimeter and redundant paths traversing large open spaces. Informal crossings are modelled where all paths intersect at streets, unless streets are wider than four lanes, or there are physical barriers or signage prohibiting crossings. Finally, in the absence of physical pedestrian infrastructure, single links are modelled on shared streets, unless streets are wider than $8 \mathrm{~m}$, in which case a separate link is modelled on either side of the roadway. These principles were employed to minimise errors when digitising pedestrian paths to ensure accuracy. Further verification took place during field visits conducted from October to December 2019 to confirm the existence and correctness of network links. Errors were further minimised by quality assurance checks and running network connectivity tools within ArcGIS. Finally, each network was used to create a network dataset using the ArcGIS Network Analyst 10.7.0.

3.3. Pedestrian Dataset. Proportionate stratified random sampling was utilised to select pedestrian segments for data collection within each $400 \mathrm{~m}$ study site. This ensured that all areas of a study site were observed but focused mainly on the core of each area. Approximately 75-100 pedestrian segments were surveyed in each site. Pedestrian counts were recorded during field surveys to each study site on weekdays during two periods: Morning (7-9 am) and Midday (11 am-1 pm). Pedestrian counts were obtained using the "snapshot" method, a technique that involves photographing the number of pedestrians on each segment at a given moment $[13,16]$. Photographs were taken approximately $10-15 \mathrm{~m}$ apart with the same section of each segment captured twice. The count for each section was then averaged between the two photographs and this was continued until the entire segment was covered. Finally, total pedestrian 
TABle 1: Pedestrian network characteristics.

\begin{tabular}{lcccc}
\hline \multirow{2}{*}{ Study site } & \multirow{2}{*}{ Network traits } & \multicolumn{2}{c}{ Pedestrian network } \\
& & Length $(\mathrm{m})$ & Links & Nodes \\
\hline Bangkok-Sukhumvit & Broken grid, cul-de-sacs, long blocks & 11,257 & 189 & 117 \\
Manila-Carriedo & Deformed grid & 18,948 & 464 & 255 \\
Osaka-Namba & Irregular grid & 28,053 & 960 & 557 \\
Taipei-Songjiang Nanjing & Regular grid & 21,236 & 674 & 409 \\
\hline
\end{tabular}

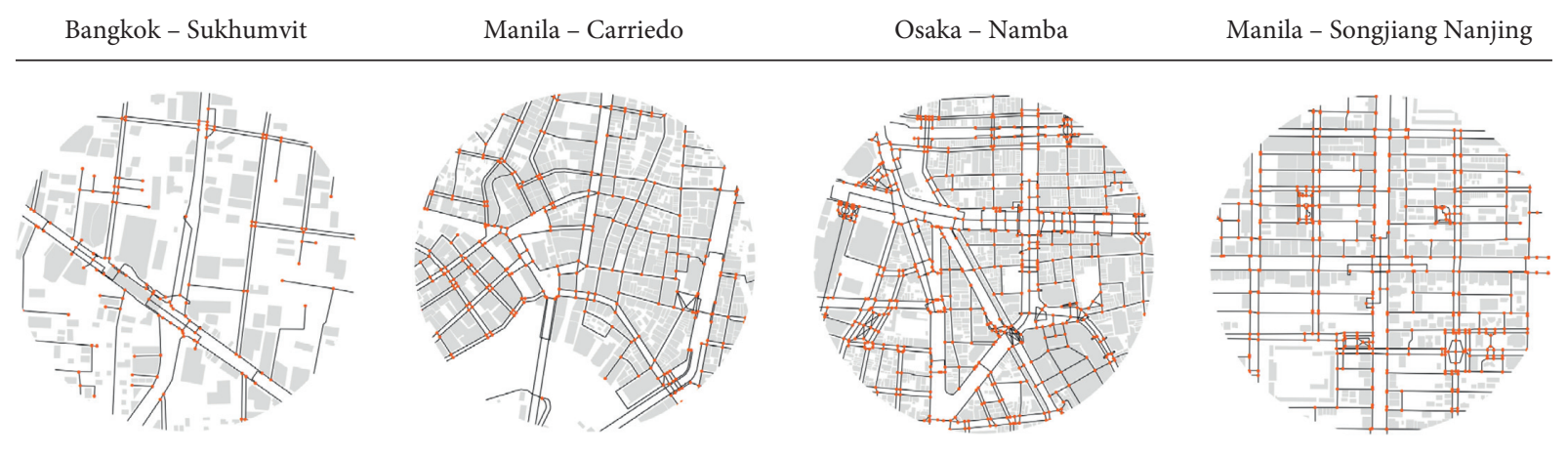

Figure 1: Spatial distribution of observed pedestrian densities $(400 \mathrm{~m})$.

counts recorded on surveyed segments were relativised by the segments length and multiplied by 100 to give a measure of how many pedestrians were encountered per $100 \mathrm{~m}$ of network [8]. This relativised pedestrian density serves as the dependent variable in this study.

This study focuses on data from the midday period. After analysing the pedestrian data, it was apparent that not only was the midday period the peak for pedestrian activity, but also it was more robust for analysis. Across all study sites, a total of 5,823 pedestrians were recorded in the morning compared to 8,820 during the midday period. However, many segments observed during the morning period recorded zero counts, resulting in too many outliers for our analysis to be meaningful. This temporal pattern is representative of urban sites that are dominated by retail land uses, which is a focus of this study. In simple terms, our selected stations are not primarily used by morning commuters and the areas become far busier during the day as pedestrians' frequent shops and restaurants. While it is desirable to include as many time periods as possible, the midday period is always included in similar pedestrian studies $[8,9,13,17]$ and some researchers have conducted analyses using their respective peak period data only [11]. Moreover, some studies [16] have specifically focused on this period when investigating similar retail-led urban neighbourhoods. Thus, we argue that the midday period is representative based on the land-use patterns and observed pedestrian activity and can be used as a reference for researchers focusing on analysing pedestrian activity in similar retail-led urban areas.

Table 2 shows that the highest number of observed pedestrians was in Manila and Osaka. The median density of moving pedestrians per $100 \mathrm{~m}$ is $31.39,12.07,9.01$, and 8.65 for Manila, Taipei, Bangkok, and Osaka, respectively, while their corresponding means are 44.36, 18.39, 14.29, and 25.46. Spatial distributions of pedestrian densities vary across all study sites (Figure 1). In Bangkok, pedestrian densities are highest on pedestrian segments closest to metro entrances located on major arterials, while in Manila and Osaka, the highest densities were observed in pedestrianised retail areas along marketed streets in Manila and along pedestrian arcades that run north-south in Osaka. In Taipei, pedestrian densities are more evenly distributed due to its gridded network, which provides a multiplicity of route options.

3.4. Selection of Variables. This study employs segment-level data in analysing the relationship between centrality and pedestrian density. In total, nine independent variables were selected: three addressing centrality, two land use, two transportation proximity, and two pedestrian level design.

Data were obtained from OSM databases and verified through field visits. Land-use data were derived from OSM point of interest (POI) data that were imported into ArcGIS 10.7 and then classified into retail, commercial, institutional, and residential land-use categories. Ground-floor proportional land-use measures were employed due to the lack of readily available parcel-level data for all study sites. These measures are based on the number of frontages at the segment level and have been shown to be significantly correlated with pedestrian activity $[8,13,14]$. Centrality measures were calculated locally up to a $400 \mathrm{~m}$ radius utilising ArcGIS Network Analyst and Urban Network Analyst (UNA), an open-source toolbox used to compute centrality measures [56]. These measures were calculated on larger $800 \mathrm{~m}$ DPNs to minimise the "edge effect" which is common in centrality analysis and results in analytical bias when imposing artificial network boundaries [57]. Each variable is explained in detail below. 

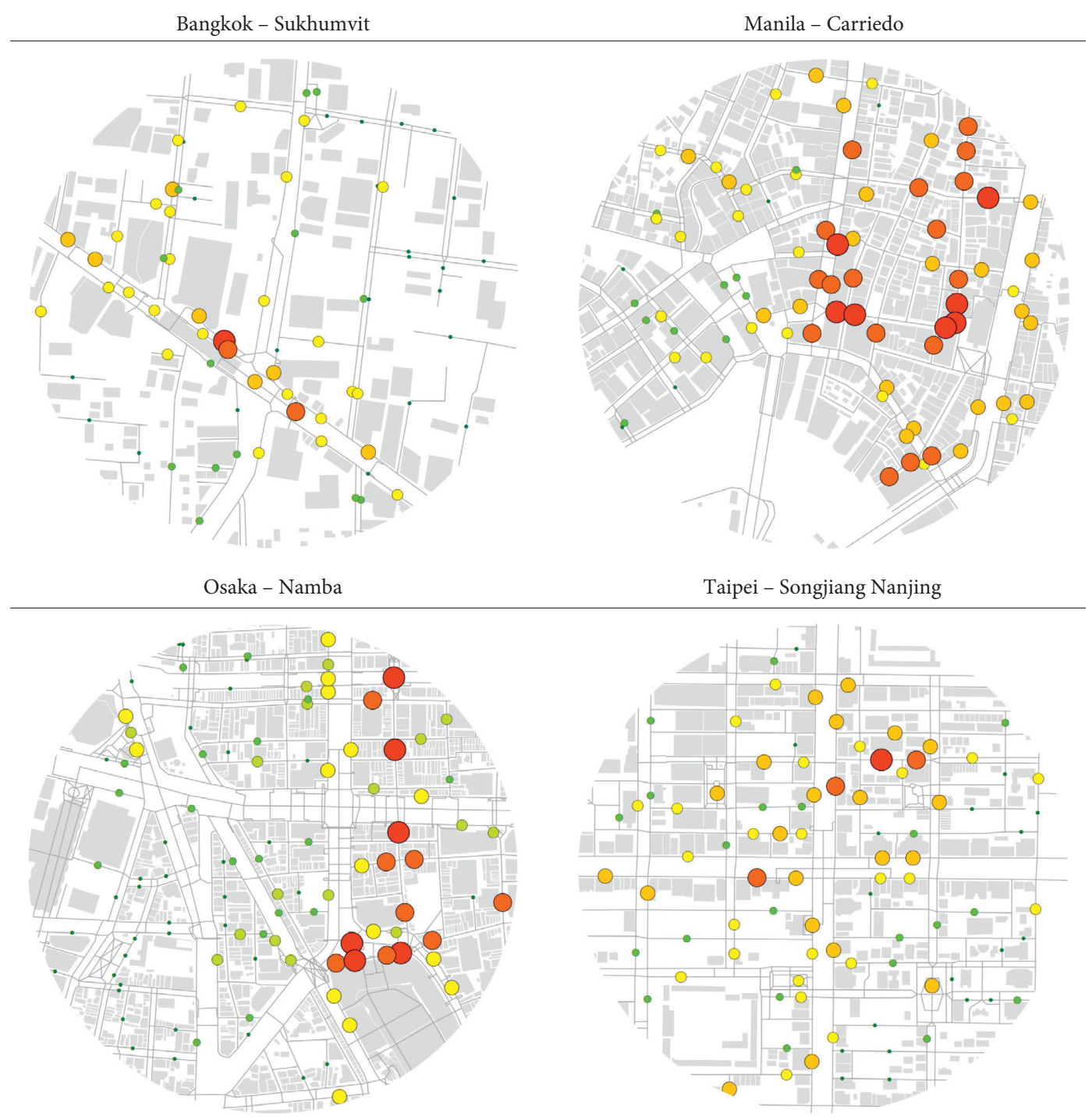

$\cdot 0-5 \quad 6-10 \quad \bigcirc 11-25 \bigcirc 26-50 \bigcirc 51-100$

$101-250+$

Pedestrians per $100 \mathrm{~m}$

Figure 2: Study site dedicated pedestrian networks (400 m).

Table 2: Pedestrian density descriptive statistics.

\begin{tabular}{|c|c|c|c|c|c|}
\hline \multirow{2}{*}{ Study sites } & \multicolumn{5}{|c|}{ Pedestrian densities per $100 \mathrm{~m}$} \\
\hline & Min & Max & Sum & Med & Mean \\
\hline Bangkok-Sukhumvit & 0.00 & 115.37 & $1,057.09$ & 9.01 & 14.29 \\
\hline Manila-Carriedo & 1.37 & 259.23 & $3,549.10$ & 31.39 & 44.36 \\
\hline Osaka-Namba & 0.00 & 225.26 & $2,596.63$ & 8.65 & 25.46 \\
\hline Taipei-Songjiang Nanjing & 1.25 & 108.82 & $1,617.06$ & 12.07 & 18.39 \\
\hline All sites & 14.29 & 44.36 & $8,819.88$ & 18.39 & 23.08 \\
\hline
\end{tabular}

(a) Betweenness Centrality. It measures the importance of an element in a network in terms of how many shortest paths pass through it [43]. Betweenness is interpreted as the probability that a person passes through a certain location on a network given all other possibilities. Normalised betweenness is defined by

$$
\mathrm{BC}(i)^{r}=\frac{2}{(N-1)(N-2)} \sum_{j, k \in G\{\{i\}, d[j, k] \leq r} \frac{n_{\mathrm{jk}}[i]}{n_{\mathrm{jk}}} .
$$

We have that $\mathrm{BC}(i)^{r}$ is the normalised betweenness of node $i$ within search radius $r ; n_{\mathrm{jk}}[i]$ is the number of network shortest paths between nodes $j$ and $k$ 
which pass through node $i$; and $n_{\mathrm{jk}}$ is the total number of shortest paths between nodes $j$ and $k$.

(b) Closeness Centrality. It measures how close an element is to all other elements in a network calculated as the mean of the shortest path lengths [44]. Closeness indicates the potential of a network location to attract movement or, in this case, pedestrian activity. Normalised closeness is defined by

$$
\mathrm{CC}(i)^{r}=\frac{N-1}{\sum_{j \in G-\{i\}, d[i, j] \leq r} d[i, j]} .
$$

We have that $\mathrm{CC}(i)^{r}$ is the normalised closeness of node $i$ within search radius $r$; and $d[i, j]$ is the shortest path distance between nodes $i$ and $j$.

(c) Straightness Centrality. It measures how closely shortest path distances between network elements resemble their corresponding Euclidean or straightline distances [42]. Straightness is interpreted as a measure of visual connectivity with higher values implying more direct and visible routes to destinations [9]. Normalised straightness is defined by

$$
\mathrm{SC}(i)^{r}=\frac{1}{N-1} \sum_{j, k \in G\{i\}, d[i, j] \leq r} \frac{d_{\mathrm{ij}} \text { Eucl }}{d_{\mathrm{ij}}} .
$$

We have that $\mathrm{SC}(i)^{r}$ is the normalised straightness of node $i$ within search radius $r$; $d_{\mathrm{ij}}^{\text {Eucl }}$ is the Euclidean distance between nodes $i$ and $j$; and $d_{\mathrm{ij}}$ is the shortest path distance between nodes $i$ and $j$.

(d) Proportion of Retail. It measures the proportion of ground-floor retail land uses to the proportion of total land uses on each surveyed segment.

(e) Proportion of Residential. It measures the proportion of ground-floor residential land uses to the proportion of total land uses on each surveyed segment.

(f) Distance to Bus Stop. It is the network distance from each surveyed segment to the nearest bus stop.

(g) Distance to Metro. It is the network distance from each surveyed segment to the nearest metro station entrance.

(h) Path Width. It is the mean width of each surveyed pedestrian path segment.

(i) Path Exclusivity. Exclusive pedestrian paths include sidewalks, arcades, pedestrian zones, walkways, and public spaces. Paths are assigned a value of 1 if exclusive or 0 if shared.

We anticipate that centrality variables will be able to explain a sizeable portion of observed pedestrian densities on DPNs when considered alone. However, when controlling for built environmental variables, the explanatory power of centrality is likely to decrease. Furthermore, results will likely vary depending on the form of each study site's DPN. For example, in denser pedestrian environments, closeness is likely to explain a greater proportion of observed pedestrian densities. However, as denser environments produce greater route choices, the explanatory power of betweenness centrality is likely to decrease. Conversely, in environments with lengthy pedestrian zones, betweenness is expected to perform well.

\section{Results}

Multivariate models were developed to examine the relationship between centrality and segment-level pedestrian density utilising DPNs. Three separate models were evaluated for each study site to analyse the impact of centrality, both alone and in combination with other built environment variables. The three models are as follows: Centrality, comprised solely of centrality metrics employed in this study; Centrality + Land Use, factoring in ground-floor land uses; and Urban Form, which introduces all remaining built environment variables analysed in this study. This threemodel approach builds on similar approaches undertaken in the literature $[8,14]$ and places the performance of centrality at the centre of the analysis. Logarithmic transformations were applied to transform the pedestrian density variable into a normal distribution. All models meet key multivariate regression assumptions, including independence of observations, linearity, and normality and exhibit no excessive multicollinearity.

Table 3 summarises mean descriptive statistics for surveyed segments in each study site. Mean betweenness is considerably higher in Bangkok (752.60) owing to it having fewer route options for pedestrians. Conversely, Osaka which has a denser network has the lowest mean betweenness value (326.30) resulting from the greater number of paths available to pedestrians through its DPN. Mean closeness is also highest in Bangkok (402.10), though the differences are less pronounced across surveyed segments in sites compared to betweenness. Similar averages are observed in all areas for straightness. Additionally, centralities are evenly dispersed around the mean in all study sites with the exception of Bangkok, where betweenness exhibited moderate positive skewness due to an outlier that was included in the final analysis. Regarding land use, surveyed segments with the highest and lowest proportion of retail land uses were observed in Manila (0.80) and Taipei (0.52), respectively. Surveyed segments with the highest proportion of residential land uses were observed in Bangkok (0.19), owing to the higher number of interior residential cul-de-sac streets in the study site. Similar levels of residential land use were also observed in Taipei (0.16). Among study sites, pedestrians have to walk furthest to a bus stop in Manila $(206 \mathrm{~m})$ which operates a system of jeepneys rather than regular buses. Respectively, Taipei had the best bus accessibility $(121.18 \mathrm{~m})$. In Bangkok, pedestrians walk on average as far as $281.72 \mathrm{~m}$ to reach a subway entrance, while the corresponding figure in Osaka was as low as $100.46 \mathrm{~m}$ for surveyed segments due to the higher number of entrances around the Osaka study site. In terms of pathlevel attributes, mean path widths were highest in Taipei $(5.93 \mathrm{~m})$ and Osaka $(5.02 \mathrm{~m})$. Finally, a similar number of exclusive pedestrian paths were surveyed in all sites, with Taipei (0.60) having the lowest proportion of surveyed 
TABle 3: Study site descriptive statistics (surveyed segments).

\begin{tabular}{|c|c|c|c|c|c|c|c|c|c|c|}
\hline \multirow{2}{*}{ Variable } & \multicolumn{2}{|c|}{ Bangkok } & \multicolumn{2}{|c|}{ Manila } & \multicolumn{2}{|c|}{ Osaka } & \multicolumn{2}{|c|}{ Taipei } & \multicolumn{2}{|c|}{ All } \\
\hline & Mean & St dev & Mean & St dev & Mean & St dev & Mean & St dev & Mean & St dev \\
\hline \multicolumn{11}{|l|}{ Centrality $(400 \mathrm{~m})$} \\
\hline Betweenness* & 752.60 & 434.30 & 405.93 & 154.91 & 326.30 & 115.85 & 356.51 & 140.43 & 444.25 & 285.64 \\
\hline Closeness** & 402.10 & 33.28 & 381.77 & 19.81 & 377.33 & 9.89 & 379.88 & 14.40 & 384.35 & 22.29 \\
\hline Straightness & 0.80 & 0.05 & 0.81 & 0.02 & 0.80 & 0.03 & 0.80 & 0.02 & 0.80 & 0.03 \\
\hline \multicolumn{11}{|c|}{ Land Use (ground-level) } \\
\hline Prop. retail & 0.66 & 0.36 & 0.80 & 0.32 & 0.64 & 0.04 & 0.52 & 0.39 & 0.65 & 0.38 \\
\hline Prop. residential & 0.19 & 0.32 & 0.03 & 0.08 & 0.04 & 0.12 & 0.16 & 0.25 & 0.10 & 0.22 \\
\hline \multicolumn{11}{|l|}{ Urban Form } \\
\hline Distance to bus & 197.51 & 128.51 & 206.00 & 86.36 & 153.38 & 93.66 & 121.18 & 55.01 & 166.87 & 98.65 \\
\hline Distance to metro & 281.72 & 128.51 & 269.30 & 124.38 & 100.46 & 67.65 & 215.29 & 112.36 & 208.97 & 140.99 \\
\hline Path width $(\mathrm{m})$ & 3.57 & 1.42 & 4.70 & 3.30 & 5.02 & 3.63 & 5.93 & 4.64 & 4.86 & 3.60 \\
\hline Prop. exclusive paths & 0.76 & 0.43 & 0.74 & 0.44 & 0.77 & 0.41 & 0.60 & 0.49 & 0.72 & 0.45 \\
\hline
\end{tabular}

${ }^{*}$ Betweenness centrality values expressed $\times 10^{4}$. ${ }^{* *}$ Closeness centrality values expressed $\times 10^{5}$.

pedestrian paths, owing to the high number of shared streets in the study site.

Multivariate regression results are reported in Tables 4-7. Each table reports unstandardised regression coefficients $(\beta)$, $t$-test scores $(t)$ used to calculate statistical significance, and standardised regression coefficients $(\operatorname{Std} \beta)$ for each independent variable.

Table 4 presents multivariate regression results for Bangkok. Across all models, centrality appears poor at explaining segment-level pedestrian density. Considered alone, centrality metrics account for only $6 \%(p<0.10)$ of observed pedestrian densities. Among significant variables, only closeness is positively associated with pedestrian density. When land-use variables are introduced, the model fit improves significantly to explain up to $46 \%(p<0.01)$ of observed pedestrian densities. In this model, pedestrian densities are overwhelmingly explained by the proportion of residential land uses, indicating that as paths become more residential in nature, pedestrian activity declines. This is the only statistically significant variable, with a standardised coefficient of -0.70 . Factoring in remaining variables results in a marginal increase of $4 \%$ in the explanatory power of the Urban Form model $(p<0.01)$. None of the path-level design attributes were found to be significantly associated with pedestrian movement densities. In this model, pedestrian densities are largely explained by metro distance and residential land uses that have significant standardised coefficients of -0.38 . The explanatory power of these variables can be attributed to network structure. As distance increases from the station, the network becomes less connected and cul-de-sacs are common deeper into the network. In these locations, residences accumulate and lower pedestrian volumes were observed. Similarly, retail uses accumulate around the station where several large shopping centres are located and decrease with distance from the station. Interestingly, it is residential and not retail land uses that are associated with pedestrian activity. This is likely explained by their moderate collinearity and how land use is strictly separated in the study site.

A more substantial relationship is revealed between centrality and segment-level pedestrian density in Manila
(Table 5). In all models, at least two centrality metrics are significant. Centrality alone explains $19 \%(p<0.01)$ of observed pedestrian densities with betweenness and closeness contributing equally to the model with significant standardised coefficients of 0.45 and -0.45 , respectively. The inclusion of land-use measures significantly improves model fit. Centrality and land use together explain $46 \%(p<0.01)$ of the variation in pedestrian activity. Retail land uses are the most important factor in the model, with a significant standardised coefficient of 0.47 . Entering the remaining Urban Form variables results in a $15 \%$ increase in the explanatory power of the model. In this model, $61 \%(p<0.01)$ of pedestrian densities are explained by a variety of variables including all centrality and path-level design measures. The most important factors are retail intensity, path width, and betweenness, which have significant standardised coefficients of $0.38,0.34$, and 0.28 , respectively. Looking at the standardised values, the proportion of retail on each segment is the biggest driver of pedestrian activity. As described earlier, a large market stretches over a sizeable portion of the site, accounting for the strong performance of retail in all models. The good performance of centrality across models, notably betweenness, is a factor of our network modelling approach. Market streets that tend to be long were modelled as a single line, reducing route options that would exist if they were modelled with a path on either side of the street. This similarly explains the negative association between closeness and pedestrian density, as streets modelled with sidewalks on either side are common to the west of the station where fewer pedestrians were observed.

In Osaka, centrality had the strongest association with segment-level pedestrian density when considered alone (Table 6). Centrality measures were found to explain as much as $24 \%(p<0.01)$ of the variation in pedestrian densities, the best performance among all study sites. Interestingly, straightness had the largest influence and is the only significant contributor with a standardised coefficient of 0.47 . Introducing land-use measures produces a smaller improvement in model fit than that seen in Bangkok and Manila. Together with centrality, they explain 36\% $(p<0.01)$ 
TABLE 4: Bangkok-Sukhumvit: multivariate regression results.

\begin{tabular}{|c|c|c|c|c|c|c|c|c|c|}
\hline \multirow{2}{*}{ Variable } & \multicolumn{3}{|c|}{ Centrality } & \multicolumn{3}{|c|}{ Centrality + LU } & \multicolumn{3}{|c|}{ Urban Form } \\
\hline & $\beta$ & $T$ & $\operatorname{Std} \beta$ & $\beta$ & $t$ & $\operatorname{Std} \beta$ & $\beta$ & $t$ & $\operatorname{Std} \beta$ \\
\hline Betweenness & -14.83 & -1.89 & $-.29^{*}$ & -1.57 & -.25 & -.03 & 5.96 & .87 & .12 \\
\hline Closeness & 2756.69 & 2.66 & $.41^{* *}$ & 1063.93 & 1.30 & .16 & -78.61 & -.08 & -.01 \\
\hline Straightness & -.04 & -.01 & -.00 & -3.68 & -.89 & -.08 & 1.91 & .35 & .04 \\
\hline Retail (prop.) & & & & -.16 & -.19 & -.03 & -.32 & -.38 & -.05 \\
\hline Residential (prop.) & & & & -4.86 & -4.92 & $-.70^{* * *}$ & -2.67 & -2.09 & $-.38^{* *}$ \\
\hline Distance to bus stop & & & & & & & -.00 & -.30 & -.04 \\
\hline Distance to metro & & & & & & & -.01 & -2.21 & $-.38^{* *}$ \\
\hline Path width & & & & & & & .09 & .55 & .06 \\
\hline Path exclusivity & & & & & & & .58 & .77 & .11 \\
\hline \multicolumn{10}{|l|}{ \# observations: 74} \\
\hline R-squared & & .09 & & & .50 & & & .56 & \\
\hline Adj. R-squared & & .06 & & & .46 & & & .50 & \\
\hline
\end{tabular}

${ }^{*} p<0.10 ;{ }^{* *} p<0.05 ;{ }^{* * *} p<0.01$.

TABLE 5: Manila-Carriedo: multivariate regression results.

\begin{tabular}{|c|c|c|c|c|c|c|c|c|c|}
\hline \multirow{2}{*}{ Variable } & \multicolumn{3}{|c|}{ Centrality } & \multicolumn{3}{|c|}{ Centrality + LU } & \multicolumn{3}{|c|}{ Urban Form } \\
\hline & $\beta$ & $t$ & $\operatorname{Std} \beta$ & $\beta$ & $t$ & $\operatorname{Std} \beta$ & $\beta$ & $t$ & $\operatorname{Std} \beta$ \\
\hline Betweenness & 30.76 & 3.86 & $.45^{* * *}$ & 20.64 & 3.07 & $.30^{* *}$ & 19.22 & 2.90 & $.28^{* *}$ \\
\hline Closeness & -2420.72 & -3.93 & $-.45^{* * *}$ & -2005.19 & -3.96 & $-.37^{* * *}$ & -1024.18 & -1.91 & $-.19^{*}$ \\
\hline Straightness & -5.78 & -1.18 & -.12 & -7.20 & -1.80 & $-.15^{*}$ & -9.03 & -2.21 & $-.19^{* *}$ \\
\hline Retail (prop.) & & & & 1.55 & 5.27 & $.47^{* * *}$ & 1.25 & 4.60 & $.38^{* * *}$ \\
\hline Residential (prop.) & & & & -2.21 & -1.87 & $-.17^{*}$ & -.46 & -.43 & -.03 \\
\hline Distance to bus stop & & & & & & & .00 & .64 & .05 \\
\hline Distance to metro & & & & & & & -.00 & -2.34 & $-.21^{* *}$ \\
\hline Path width & & & & & & & .11 & 3.71 & $.34^{* * *}$ \\
\hline $\begin{array}{l}\text { Path exclusivity } \\
\text { \# observations: } 80\end{array}$ & & & & & & & .56 & 2.98 & $.23^{* *}$ \\
\hline R-squared & & .22 & & & .50 & & & .65 & \\
\hline Adj. R-squared & & .19 & & & .46 & & & .61 & \\
\hline
\end{tabular}

${ }^{*} p<0.10 ;{ }^{* *} p<0.05 ;{ }^{* * *} p<0.01$.

TABLE 6: Osaka-Namba: multivariate regression results.

\begin{tabular}{|c|c|c|c|c|c|c|c|c|c|}
\hline \multirow{2}{*}{ Variable } & \multicolumn{3}{|c|}{ Centrality } & \multicolumn{3}{|c|}{ Centrality + LU } & \multicolumn{3}{|c|}{ Urban Form } \\
\hline & $\beta$ & $t$ & $\operatorname{Std} \beta$ & $\beta$ & $t$ & $\operatorname{Std} \beta$ & $\beta$ & $t$ & $\operatorname{Std} \beta$ \\
\hline Betweenness & 16.19 & 1.07 & .11 & 12.07 & .86 & .08 & 5.56 & .45 & .04 \\
\hline Closeness & -775.11 & -.45 & -.05 & -176.85 & -.11 & -.01 & -320.21 & -.24 & -.02 \\
\hline Straightness & 30.67 & 4.40 & $.47^{* * *}$ & 25.52 & 3.92 & $.39^{* * *}$ & 19.52 & 3.15 & $.30^{* *}$ \\
\hline Retail (prop.) & & & & 1.12 & 3.07 & $.26^{* *}$ & 1.60 & 4.79 & $.38^{* * *}$ \\
\hline Residential (prop.) & & & & -2.69 & -2.34 & $-.20^{* *}$ & .10 & .09 & $.01^{* *}$ \\
\hline Distance to bus stop & & & & & & & -.00 & -2.15 & $-.20^{* *}$ \\
\hline Distance to metro & & & & & & & -.00 & -.65 & -.06 \\
\hline Path width & & & & & & & .17 & 5.06 & $.37^{* * *}$ \\
\hline Path exclusivity & & & & & & & .79 & 2.43 & $.19^{* *}$ \\
\hline \# observations: 102 & & & & & & & & & \\
\hline R-squared & & .26 & & & .39 & & & .58 & \\
\hline Adj. R-squared & & .24 & & & .36 & & & .54 & \\
\hline
\end{tabular}

${ }^{*} p<0.10 ;{ }^{* *} p<0.05 ;{ }^{* * *} p<0.01$.

of observed pedestrian densities. In this model, land-use variables behave as expected with retail and residential land uses being positively and negatively associated with pedestrian density, respectively. Notably, straightness remains the strongest contributor, with a significant standardised coefficient of 0.39 . Factoring in remaining variables results in a considerable improvement in the explanatory power of the model. The Urban Form model explains 54\% $(p<0.01)$ of observed pedestrian densities. Path-level attributes enter the model as significant contributors of pedestrian activity. The 
TABLE 7: Taipei-Songjiang Nanjing: multivariate regression results.

\begin{tabular}{|c|c|c|c|c|c|c|c|c|c|}
\hline \multirow{2}{*}{ Variable } & \multicolumn{3}{|c|}{ Centrality } & \multicolumn{3}{|c|}{ Centrality + LU } & \multicolumn{3}{|c|}{ Urban Form } \\
\hline & $\beta$ & $t$ & Std $\beta$ & $\beta$ & $t$ & $\operatorname{std} \beta$ & $\beta$ & $t$ & Std $\beta$ \\
\hline Betweenness & 25.68 & 2.97 & $.38^{* *}$ & 14.63 & 1.83 & $.21^{*}$ & 11.63 & 1.67 & $.17^{*}$ \\
\hline Closeness & -600.92 & -.79 & -.09 & 48.70 & .07 & .01 & -1127.21 & -1.66 & -.17 \\
\hline Straightness & 5.85 & 1.10 & .14 & 4.07 & .86 & .10 & 9.54 & 2.04 & $.23^{* *}$ \\
\hline Retail (prop.) & & & & .78 & 3.24 & $.31^{* *}$ & .78 & 3.50 & $.31^{* *}$ \\
\hline Residential (prop.) & & & & -.94 & -2.48 & $-.24^{* *}$ & -.15 & -.41 & -.04 \\
\hline Distance to bus stop & & & & & & & .00 & -.26 & -.02 \\
\hline Distance to metro & & & & & & & -.00 & -4.04 & $-.37^{* * *}$ \\
\hline Path width & & & & & & & .06 & 3.37 & $.27^{* *}$ \\
\hline Path exclusivity & & & & & & & .20 & 1.11 & .10 \\
\hline \multicolumn{10}{|l|}{ \# observations: 88} \\
\hline R-squared & & .19 & & & .38 & & & .56 & \\
\hline Adj. R-squared & & .16 & & & .34 & & & .51 & \\
\hline
\end{tabular}

${ }^{*} p<0.10 ;{ }^{* *} p<0.05 ;{ }^{* * *} p<0.01$.

most important factors are retail, path width, and straightness with significant standardised coefficients of $0.38,0.37$, and 0.30 , respectively. These results indicate that pedestrians orient themselves in terms of retail, design attributes, and visual connectivity. The Osaka site is highly pedestrianised in nature. Ground-floor retail land uses are consistent throughout, and the area is characterised by numerous wide arcades and pedestrian zones that attract large numbers of shoppers. Noteworthy is how Osaka is the only site where metro distance has no significant influence on pedestrian activity. Metro station entrances are dispersed throughout the area, meaning that distances are fairly uniform, nullifying the effectiveness of this variable.

Table 7 presents multivariate regression results for Taipei. Focusing on centrality, we can see that centrality alone can explain $16 \%(p<0.01)$ of observed pedestrian densities. Among centrality variables, the only significant correlate of pedestrian density is betweenness with a standardised regression coefficient of 0.38 . Land-use measures enter the model as significant variables and perform as expected. Centrality and land use together explain $34 \%$ $(p<0.01)$ of the variation in pedestrian activity. In this model, the strongest associations are between retail, residential, and betweenness with standardised regression coefficients of $0.31,-0.21$, and 0.21 , respectively. When all variables are entered into the Urban Form model, the model fit improves to explain up to $51 \%(p<0.01)$ of observed pedestrian densities. The largest contributors to pedestrian movement densities are metro distance, retail, and path width with standardised coefficients of $-0.37,0.31$, and 0.27 , respectively. Additionally, both straightness and betweenness are significant when controlling for all Urban Form variables with standardised coefficients of 0.23 and 0.17 , respectively. Among all sites, Taipei has the highest mean path widths recorded due to large sidewalks fronting main streets that are retail-heavy and close to metro station entrances. It is in these locations in addition to the several pedestrian zones to the northeast of the site where pedestrian densities are highest, explaining the strong associations between these variables.

\section{Discussion}

The present study confirms that centrality measures derived from DPNs are associated with increased pedestrian activity. Results indicate that centrality alone can explain $6-24 \%$ of segment-level pedestrian density in station environments in Asian cities. Excluding Bangkok, this figure increases to 16-24\%. These ranges are comparatively lower than those cited in earlier Space Syntax studies that employ either axial or segmental approaches to urban street networks $[12,28,40,48]$. Naturally, DPNs are far larger and complex than street centreline networks. They consist of more links and nodes, resulting in shorter distances between network elements, which in turn impacts centrality. This further varies with the complexity inherent in each urban network form. Seemingly, centrality is more strongly associated with pedestrian activity in denser networks. This is confirmed by the weaker associations observed in Bangkok, which consists of fewer network elements with greater distances between them.

The influence of centrality is somewhat diminished when we control for the built environment. Still, excluding Bangkok, centrality remains a substantial contributor to pedestrian activity. In line with similar street network centrality studies, our Urban Form models suggest that the primary factors in explaining pedestrian densities are the proportion of ground-floor land uses, chiefly retail uses, proximity to rail transit, and path width [8, 9, 14, 58, 59]. Notably, path exclusivity was only significant in Manila and Osaka. This is likely due to the high number of shared streets within Asian cities that result in pedestrians being somewhat more used to walking within close proximity of traffic.

An important consideration is the performance of individual centrality measures. Looking at our results, different metrics had stronger relationships in different sites. For example, betweenness performed particularly well in Manila and straightness in Osaka. Existing literature points to closeness or integration as being the key metric in explaining pedestrian activity on street networks. Generally, closeness was weakly associated with pedestrian density; and when an 
association was found, as in Manila, it was negative. The performance of centrality across our target cities is a factor of two things: our network modelling approach and the spatial variance of centralities within each study site. In DPNs that consist of long pedestrian zones and shared paths modelled as single lines, betweenness performs strongly. Conversely, in dense DPNs with more paths modelled on either side of a street increasing route options, the explanatory power of betweenness decreases. Unexpectedly, in denser DPNs, straightness performs best due to reduced straight-line distances between nodes indicating that pedestrians in these environments orient themselves strongly in terms of visual connectivity. This is best exemplified by Osaka, where pedestrian activity is higher on segments that are more visually connected with director routes to destinations. These segments were typically pedestrian arcades and shopping zones, where pedestrian activity was notably higher. Naturally, the spatial characteristics of variables vary within each study site, as well as in comparison with each city. For example, segments with high betweenness values in Manila are concentrated to the east of the station and correspond strongly to pedestrian market streets. Similarly, the visually connected segments with high straightness values in Osaka were located to the northeast of the station, running in a northsouth orientation. These locations where the highest pedestrian numbers were observed raise the question concerning whether it is the centrality of the DPN that is influencing the location and distribution of retail land uses which ultimately promotes pedestrian activity. The spatial variance of these variables over larger areas is an important consideration noted in other studies [60]. However, due to the relatively small size of the study sites presented in this study, the importance of this is difficult to ascertain.

It is worth recalling that centrality is a multifaceted concept with numerous indices available depending on how the notion of "being central" is defined. Porta et al. [10] proposed the use of Multiple Centrality Analysis based on multiple indices to provide clarity in studies employing centrality approaches. This study took a similar approach with the inclusion of three common indices. Evidently, further research is needed to provide greater clarity to our research findings.

\section{Conclusions}

This study builds on existing studies by applying centrality approaches to DPNs. Our findings imply that centrality measures are useful as a descriptive measure to explain pedestrian movement variations when derived from DPNs with minimal data requirement. This application is useful in countries where other built environment data is less complete or unavailable. Ultimately, the role that centrality plays is secondary when other built environment factors are considered. Results indicate that pedestrians orient themselves more strongly to retail attractors, proximity to rail transit, and path width, rather than solely the spatial structure of the DPN. Thus, practitioners pursuing pedestrian friendly environments should focus on promoting retail and transit accessibility and wider pedestrian exclusive paths. Dense, well-connected networks should also be pursued to encourage further accessibility and connectivity. On this point, pedestrian network centrality can contribute to planning more pedestrian-centric cities by further understanding how people move and interact with the pedestrian environment. This is important for greater understanding of how cities should be designed to better accommodate pedestrian movement patterns.

We identify several areas in which the present study could be improved to allow more concrete conclusions to be made. First, while OpenStreetMap (OSM) network data is helpful in creating DPNs, it is still a time-consuming process. As a result, our analytical focus was limited to $400 \mathrm{~m}$. Centrality metrics are typically applied to larger networks than those used in our study, and our findings may be limited by the extent of our networks. Larger DPNs should be considered in future studies in different urban environments to help provide clarity to the effectiveness of centrality derived from DPNs. Finally, while the snapshot method of recording pedestrians served its purpose, collecting pedestrian data over longer time periods at different times of day would likely provide more robust results. Nevertheless, this research lays a solid foundation for further investigation in this area with methods that can be applied to other regions globally.

\section{Data Availability}

The data used to support the findings of this study are available from the corresponding author upon request.

\section{Conflicts of Interest}

The authors declare that there are no conflicts of interest regarding the publication of this article.

\section{References}

[1] H. Zhang and Y. Zhang, "Pedestrian network analysis using a network consisting of formal pedestrian facilities: sidewalks and crosswalks," Transportation Research Record: Journal of the Transportation Research Board, vol. 2673, no. 7, pp. 294-307, 2019.

[2] D. M. Pearce, R. Matsunaka, and T. Oba, "Comparing accessibility and connectivity metrics derived from dedicated pedestrian networks and street networks in the context of asian cities," Asian Transport Studies, vol. 7, pp. 1-10, 2021.

[3] G. Tal and S. Handy, "Measuring n accessibility and connectivity in a robust pedestrian network," Transportation Research Record: Journal of the Transportation Research Board, vol. 2299, no. 1, pp. 48-56, 2012.

[4] S. M. Cruise, R. F. Hunter, F. Kee, M. Donnelly, G. Ellis, and M. A. Tully, "A comparison of road- and footpath-based walkability indices and their associations with active travel," Journal of Transport \& Health, vol. 6, pp. 119-127, 2017.

[5] G. Ellis, R. F. Hunter, M. A. Tully, M. Donnelly, L. Kelleher, and F. Kee, "Connectivity and physical activity: using footpath networks to measure the walkability of built environments," Environment and Planning B: Planning and Design, vol. 43, no. 1, pp. 130-151, 2016. 
[6] R. Cervero and K. Kockelman, "Travel demand and the 3Ds: density, diversity, and design," Transportation Research Part D: Transport and Environment, vol. 2, no. 3, pp. 199-219, 1997.

[7] C. Lee and A. V. Moudon, “The 3D's+R: quantifying land use and urban form correlates of walking," Transportation Research Part D: Transport and Environment, vol. 11, pp. 204215, 2006.

[8] A. Özbil, T. Gurleyen, D. Yesiltepe, and E. Zunbuloglu, "Comparative associations of street network design, streetscape attributes and land-use characteristics on pedestrian flows in peripheral neighbourhoods," International Journal of Environmental Research and Public Health, vol. 16, no. 1846, pp. 1-23, 2019.

[9] C. D. Kang, "The S +5Ds: spatial access to pedestrian environments and walking in seoul, korea," Cities, vol. 77, pp. 130-141, 2018.

[10] S. Porta, P. Crucitti, and V. Latora, "Multiple centrality assessment in parma: a network analysis of paths and open spaces," Urban Design International, vol. 13, no. 1, pp. 41-50, 2008.

[11] A. Hajrasouliha and L. Yin, "The impact of street network connectivity on pedestrian volume," Urban Studies, vol. 52, no. 13, pp. 2483-2497, 2015.

[12] B. Hillier and S. Iida, "Network effects and psychological effects: a theory of urban movement," in Proceedings of the Fifth International Space Syntax Symposium, pp. 553-564, Techne Press, Delft Netherlands, 13 June 2005.

[13] E. Choi and D. Koch, "Movement and the connectivity of streets: a closer look at route distribution and pedestrian density,"vol. 65, pp. 1-65, in Proceedings of the 10th International Space Syntax Symposium, vol. 65, pp. 1-65, UCL, London, England, 13-July 2015.

[14] A. Özbil and J. Peponis, "Modelling street connectivity and pedestrian movement according to standard GIS street network representations," in Proceedings of the 6th International Space Syntax Symposium, p. 018, ITU faculty of Architecture, Istanbul, Turkey, 12 June 2007.

[15] Y. Lerman, Y. Rofè, and I. Omer, "Using space Syntax to model pedestrian movement in urban transportation planning," Geographical Analysis, vol. 46, pp. 392-410, 2014.

[16] K. Fang, X. Wang, L. Chin, X. Zhang, and N. Furuya, "Research on the correlation between pedestrian density and street spatial characteristics of commercial blocks in downtown area: a case study on shanghai tianzifang," Architecture and Building Engineering, vol. 18, no. 3, pp. 233-246, 2019.

[17] S. Law and M. Traunmueller, "Off the shortest path: betweenness on street network level to study pedestrian movement," in Proceedings of the IEEE 20th International Conference on Intelligent Transportation Systems (ITSC): Workshop, pp. 971-976, IEEE, Yokohama, Japan, 16 October 2017.

[18] R. C. Brownson, C. M. Hoehner, K. Day, A. Forsyth, and J. F. Sallis, "Measuring the built environment for physical activity: state of the science," American Journal of Preventive Medicine, vol. 36, pp. S99-S123, 2009.

[19] L. D. Frank and G. Pivo, "Impacts of mixed use and density on utilization of three modes of travel: single-occupant vehicle, transit, and walking," Transportation Research Record, vol. 1466, pp. 44-52, 1994.

[20] H. Badland and G. Schofield, "Transport, urban design and physical activity: an evidence-based update," Transportation Research Part D: Transport and Environment, vol. 10, no. 3, pp. 177-196, 2005.
[21] A. E. Bauman and F. C. Bull, Environmental Correlates of Physical Activity and Walking in Adults and Children: A Review of Reviews, National Institute for Health and Care Excellence (NICE), London, England, 2007.

[22] B. E. Saelens and S. Handy, "Built environment correlates of walking: a review," Medicine \& Science in Sports \& Exercise, vol. 40, no. 7, pp. S550-S566, 2008.

[23] R. Ewing and R. Cervero, "Travel and the built environment," Journal of the American Planning Association, vol. 76, no. 3, pp. 265-294, 2010.

[24] S. Handy and K. Clifton, "Evaluating neighborhood accessibility: possibilities and practicalities," Journal of Transportation and Statistics, vol. 4, no. 2, pp. 67-78, 2001.

[25] P. Hess, A. Moudon, M. Snyder, and K. Stanilov, "Site design and pedestrian travel," Transportation Research Record, vol. 1674, pp. 9-19, 1999.

[26] J. Kerr, L. Frank, J. F. Sallis, and J. Chapman, "Urban form correlates of pedestrian travel in youth: differences by gender, race-ethnicity and household attributes," Transportation Research Part D: Transport and Environment, vol. 12, no. 3, pp. 177-182, 2007.

[27] A. Forsyth, M. Oakes, K. Schmitz, and M. Hearst, "Does residential density increase walking and other physical activity?" Urban Studies, vol. 44, no. 4, pp. 679-697, 2007.

[28] A. Özbil, J. Peponis, and B. Stone, "Understanding the link between street connectivity, land use and pedestrian flows," Urban Design International, vol. 16, no. 2, pp. 125-141, 2011.

[29] N. Humpel, N. Owen, and E. Leslie, "Environmental factors associated with adults' participation in physical activity: a review," American Journal of Preventive Medicine, vol. 22, no. 3, pp. 188-199, 2002.

[30] M. J. Duncan, J. C. Spence, and W. K. Mummery, "Perceived environment and physical activity: a meta-analysis of selected environmental characteristics," International Journal of Behavioral Nutrition and Physical Activity, vol. 2, no. 11, pp. 1-9, 2005.

[31] J. Van Cauwenberg, V. Van Holle, D. Simons et al., "Environmental factors influencing older adults' walking for transportation: a study using walk-along interviews," International Journal of Behavioral Nutrition and Physical Activity, vol. 9, no. 85, pp. 1-11, 2012.

[32] K. R. Evenson, D. Sotres-Alvarez, A. H. Herring, L. Messer, B. A. Laraia, and D. A. Rodríguez, "Assessing urban and rural neighborhood characteristics using audit and GIS data: derivation and reliability of constructs," International Journal of Behavioral Nutrition and Physical Activity, vol. 6, no. 4, pp. 1-16, 2009.

[33] T. Pikora, B. Giles-Corti, F. Bull, K. Jamrozik, and R. Donovan, "Developing a framework for assessment of the environmental determinants of walking and cycling," Social Science \& Medicine, vol. 56, no. 8, pp. 1693-1703, 2003.

[34] R. Ewing, M. Connors, A. Hajrasouliha et al., Validating Urban Design Measures, Transportation Research Board 92nd Annual Meeting, Washington DC, January 2013.

[35] X. Zhang, S. Melbourne, C. Sarkar, A. Chiaradia, and C. Webster, "Effects of green space on walking: does size, shape and density matter?" Urban Studies, vol. 57, no. 16, pp. 3402-3420, 2020.

[36] P. K. Maghelal and C. J. Capp, "Walkability: a review of existing pedestrian indices," URISA Journal, vol. 23, no. 2, pp. 5-19, 2011. 
[37] D. Berrigan, L. Pickle, and J. Dill, "Associations between street connectivity and active transportation," International Journal of Health Geographics, vol. 9, no. 1, pp. 1-18, 2010.

[38] A. V. Moudon, C. Lee, A. D. Cheadle et al., "Attributes of environments supporting walking," American Journal of Health Promotion, vol. 21, no. 5, pp. 448-459, 2007.

[39] S. Handy, R. G. Paterson, and K. Butler, Planning for Street Connectivity: Getting from Here to There, American Planning Association, Planning Advisory Service, Chicago, 2003.

[40] B. Hillier, A. Penn, J. Hanson, T. Grajewski, and J. Xu, "Natural movement: or, configuration and attraction in urban pedestrian movement," Environment and Planning B: Planning and Design, vol. 20, no. 1, pp. 29-66, 1993.

[41] B. Hillier and L. Vaughan, "The city as one thing," Progress in Planning, vol. 67, no. 3, pp. 205-230, 2007.

[42] S. Porta, S. Crucitti, and V. Latora, "The network analysis of urban streets: a primal approach," Environment and Planning B: Planning and Design, vol. 33, pp. 705-725, 2006.

[43] L. Freeman, "A set of measures of centrality based on betweenness," Sociometry, vol. 40, pp. 35-41, 1977.

[44] L. Freeman, "Centrality in social networks: conceptual clarification," Social Networks, vol. 1, no. 3, pp. 215-239, 1979.

[45] S. Law, A. Chiaradia, and C. Schwander, "Towards a multimodal space Syntax analysis," in Proceedings of the: 8th International Space Syntax Symposium Santiago, pp. 1-20, PUC, Chile, South America, 3 January 2012.

[46] B. Hiller, T. Yang, and A. Turner, "Normalising least angle choice in depthmap and how it opens up new perspectives on the global and local analysis of city space," Journal of Space Syntax, vol. 3, no. 2, pp. 155-193, 2012.

[47] A. Turner, "From axial to road-centre lines: a new representation for space Syntax and a new model of route choice for transport network analysis," Environment and Planning B: Planning and Design, vol. 34, no. 3, pp. 539-555, 2007.

[48] A. Penn, B. Hillier, D. Banister, and J. Xu, "Configurational modeling of urban movement networks," Environment \& Planning (B): Planning \& Design, vol. 25, pp. 59-84, 1988.

[49] S. Read, "Space Syntax and the Dutch city," Environment and Planning B: Planning and Design, vol. 26, pp. 251-264, 1999.

[50] J. Peponis, S. Bafna, and Z. Zhang, "The connectivity of streets: reach and directional distance," Environment and Planning $B$ : Planning and Design, vol. 35, no. 5, pp. 881-901, 2008.

[51] P. J. Lamíquiz and J. López-Domínguez, "Effects of built environment on walking at the neighbourhood scale. A new role for street networks by modelling their configurational accessibility?" Transportation Research Part A, vol. 74, pp. 148-163, 2015.

[52] E. Choi and S. Sardari Sayyar, "Urban diversity and pedestrian behaviour - refining the concept of land-use for walkability," in Proceedings of the: Eighth International Space Syntax Symposium, pp. 1-15, PUC, Santiago, Chile, 3 January 2012.

[53] O. Özer and A. S. Kubat, "Walking initiatives: a quantitative movement analysis," in Proceedings of the, 6th International Space Syntax Symposium, pp. 1-16, ITU, Istanbul, Turkey, 12 January 2007.

[54] S. Fukuyama and E. Hato, "Network analysis of plaza-street system based on the historical development process of the old city of barcelona in considering the range of walking distance," Journal of Japan Society of Civil Engineers, vol. 68, no. 1, pp. 13-25, 2012, (In Japanese).

[55] United Nations Development Programme, Statistical Update: Human Development Indices and Indicators, United Nations, New York, 2018.
[56] A. Sevtsuk and M. Mekonnen, "Urban network analysis: a new toolbox for ArcGIS," International Journal of Geomatics and Spatial Analysis, vol. 22, no. 2, pp. 287-305, 2012.

[57] J. Gil, "Street network analysis "edge effects": examining the sensitivity of centrality measures to boundary conditions," Environment and Planning B: Urban Analytics and City Science, vol. 44, no. 5, pp. 819-836, 2017.

[58] J. Peponis, E. Hadjinikolaou, C. Livieratos, and D. A. Fatouros, "The spatial core of urban culture," Ekistics, vol. 56, no. 334/335, pp. 43-55, 1989.

[59] J. Peponis, C. Ross, and M. Rashid, "The structure of urban space, movement and Co-presence: the case of Atlanta," Geoforum, vol. 28, no. 3-4, pp. 341-358, 1997.

[60] S. Li, D. Lyu, G. Huang et al., "Spatially varying impacts of built environment factors on rail transit ridership at station level: a case study in guangzhou, China," Journal of Transport Geography, vol. 82, no. 102631, pp. 1-14, 2020. 